\title{
Objective measurement of sedentary behavior: impact of non-wear time rules on changes in sedentary time
}

Xanne Janssen ${ }^{1 *}$, Laura Basterfield², Kathryn N. Parkinson², Mark S. Pearce ${ }^{3}$, Jessica K. Reilly², Ashley J. Adamson², John J. Reilly ${ }^{1}$ and the Gateshead Millennium Study Core Team

\begin{abstract}
Background: Accelerometry non-wear time rules might affect sedentary time, and the associations with health outcomes such as adiposity. However, the exact effect of different non-wear time rules on sedentary time and reported changes in sedentary time is unknown. This study evaluated the effect of different accelerometry non-wear time rules on sedentary time and changes in sedentary time from age 9-12 years.
\end{abstract}

Methods: Accelerometry data were collected as part of the Gateshead Millennium Birth Cohort study. Participants were $9.3( \pm 0.4)$ years at baseline $(n=517)$ and $12.5( \pm 0.3)$ years at follow-up $(n=440)$. Sedentary time was defined using an accelerometry cut-point of 25 counts per $15 \mathrm{~s}$. Non-wear time was defined using manual data reduction (the reference method) and $10 \mathrm{~min}, 20 \mathrm{~min}$ and $60 \mathrm{~min}$ consecutive zeros. Differences between methods were analyzed using repeated measures ANOVA with Bonferroni post-hoc analyses.

Results: Mean daily sedentary time at age 9 ranged from 364 min per day to 426 min using the 10 min and 60 min rule, respectively $(p<0.05)$. At 12 years, mean daily sedentary times ranged from 424 min to 518 min $(p<0.05)$. Mean changes in daily sedentary time over the three years ranged from 60 min to 93 min using the $10 \mathrm{~min}$ and $60 \mathrm{~min}$ rule, respectively $(p<0.05)$. When adjusting for wear time, differences in average sedentary time between methods decreased from $62 \mathrm{~min}$ to $27 \mathrm{~min}$ (age 9), $95 \mathrm{~min}$ to $32 \mathrm{~min}$ (age 12) and $33 \mathrm{~min}$ to $10 \mathrm{~min}$ (changes between 9 to 12 years).

Conclusions: Using different non-wear time rules results in significant differences in daily sedentary time and changes in sedentary time. Correcting for wear time appears to be a reasonable approach to limiting these differences and may improve comparability between future studies. Using the 20 min rule, while correcting for wear time, provided the most accurate estimates of sedentary time and changes in sedentary time, compared to the manual reference in 9-12 year-olds.

Keywords: Accelerometry, Child, Adolescent, Sedentary behavior, Non-wear time

\section{Background}

There is now substantial interest in the measurement of time spent sedentary (defined as any sitting behavior with an energy expenditure value $\leq 1.5$ METs [1]) by children and adolescents, because of the potential for short and long-term health impacts of sedentary time [2], and interventions to modify sitting behavior in children are now

\footnotetext{
* Correspondence: xanne.janssen@strath.ac.uk

${ }^{1}$ School of Psychological Sciences and Health, University of Strathclyde, Glasgow, Scotland G1 1QN, UK

Full list of author information is available at the end of the article
}

underway [3]. Accelerometry is the method of choice for most researchers objectively measuring free-living physical activity and sedentary behavior, including sitting. However, from the onset of choosing accelerometry, several methodological decisions have to be made by the researcher. After the data have been collected, decisions on the definition of non-wear time, minimum wear time to define a valid day, minimum number of valid days and accelerometry cut-points need to be made and all have potential to affect the outcome variables $[4,5]$. 
To date the effect of different cut-points, minimum wear time and minimum number of valid days on physical activity and sedentary behavior outcomes have been examined $[6,7]$. However, much less is known about the effect of non-wear time criteria on sedentary time and reported changes of sedentary time. Non-wear time is often defined as counts being equal to, or greater than, a certain amount of consecutive zeros, with only a few studies using selfreport diaries to extract non-wear time [8-11]. It has been shown that different non-wear time definitions result in differences in total wear time and the number of nonwear periods [12]. In addition, as sitting often results in zero counts and is most likely to occur in bouts, rather than sporadic episodes, the chosen length of consecutive zeros may also influence the amount of sedentary time reported. Thus far, consecutive zero rules to identify and remove non-wear time range from $10 \mathrm{~min}$ of consecutive zeros to $180 \mathrm{~min}$, with or without allowed interruptions and/or using sophisticated algorithms [13, 14]. Consequently, the exact amount of time children and adolescents spend sedentary remains unclear. In addition, using inconsistent rules makes comparison of sedentary time, and changes in sedentary time, between studies very difficult [15].

Accelerometry non-wear time rules might affect sample size and sedentary time, and their associations with health outcomes such as adiposity in different ways $[14,16]$. However, the impact of non-wear time rules on sedentary time remains uncertain. Given a probable increase in sedentary time over age [17-19], any impact of non-wear time rules may become even more apparent when looking at changes in sedentary behavior during childhood and adolescence. In addition, it has been shown that patterns of sedentary behavior are different between age groups. Older children and adolescents appear to engage in longer bouts of sedentary behavior compared to younger children $[9,18,20]$. Therefore, longitudinal studies using very stringent rules to identify and remove 'non-wear time' (e.g. $10 \mathrm{~min}$ of consecutive zeros [8]) may underestimate changes in sedentary time by misclassifying it to a greater degree as nonwear time at follow up. Studies using less stringent rules to define non-wear time (e.g. $60 \mathrm{~min}$ of consecutive zeros [9]) on the other hand may overestimate the change in sedentary time. Therefore, the aims of this study were to examine the effect of different approaches to defining non-wear time on sedentary time and changes in sedentary time, and to examine the impact of correcting for wear-time on these differences.

\section{Methods}

Participants were part of the Gateshead Millennium Study birth cohort. Details of this cohort study have been published previously [21]. Participants who had not opted out of the study were contacted to take part in follow up measurements in 2008 and 2010. Measurements for the present study were collected when children were 8-10 years (from here on referred to as age 9) and 11-13 years (from here on referred to as age 12). The study was approved by the Newcastle University Faculty of Medical Sciences Ethics Committee. Informed written consent was obtained from main carer of each child, and children provided their assent to participation.

Children's height and weight were measured using standardized procedures. Height was measured to the nearest $0.1 \mathrm{~cm}$ using a Leicester portable stadiometer (Chasmors, London, United Kingdom). Weight was measured to the nearest $0.1 \mathrm{~kg}$ while wearing light clothing using a calibrated electronic scale (Tanita TBF300MA, Chasmors, London, United Kingdom). Body mass index (BMI) was calculated and UK population reference data was used to calculate the BMI z-score for each child [22].

Sedentary behavior was measured using an ActiGraph GT1M (ActiGraph Corporation; Pensacola, Florida) accelerometer. The ActiGraph is a small uni-axial accelerometer which provides valid measures of physical activity and sedentary behavior [23, 24]. Participants were asked to wear the accelerometer on the right hip during all waking hours for 7 days and only remove the monitor during water-based activities, as described previously [11, 25]. Participants and their parents were also asked to record times when the monitor was taken off using a provided $\log$ sheet, and briefly note the reasons why the devices were taken off. Data were collected in $15 \mathrm{~s}$ epochs and included in the analyses if participants had at least three days with $6 \mathrm{~h}$ per day of accelerometry data (more than $80 \%$ of the participants had more than $10 \mathrm{~h}$ per day), as a previous study suggested that this provided acceptable reliability for measurement of physical activity and sedentary behavior [26]. In the present study, epochs were defined as sedentary when recorded counts were $\leq 25$ counts per $15 \mathrm{~s}$. This Actigraph cut-point has been widely used to define sedentary behavior in adults and children, and has shown good agreement with a posture based monitor when measuring sitting in one study of children [27]. Data between $07.00 \mathrm{~h}$ and $23.59 \mathrm{~h}$ were used and possible nonwear time during this period was determined using four different rules:

1. Manual screening using the times reported in the self-report diary [11]

2. A sequence of $10 \mathrm{~min}$ or more of consecutive zeros [8].

3. A sequence of $20 \mathrm{~min}$ or more of consecutive zeros [18].

4. A sequence of $60 \mathrm{~min}$ or more of consecutive zeros [9]

Manual data reduction of all accelerometry records took place by comparing non-wear periods (i.e. a string 
of consecutive zeros) between $07.00 \mathrm{~h}$ and $23.59 \mathrm{~h}$ with the completed log sheets. Periods of consecutive zeros which were identified as non-wear time according to the log sheets and visually confirmed by a trained researcher were excluded. For the three rules using consecutive strings of zeros, data were excluded if a string of consecutive zeros was equal to or larger than 10,20 or $60 \mathrm{~min}$. No interruptions were allowed within these time periods.

A custom Microsoft Excel macro was used to calculate sedentary time per day, and the percentage of sedentary time per day for each of the four non-wear time rules. Sedentary time was corrected for wear time differences using the percentage of monitored time which was sedentary time as well as sedentary time in minutes per $12 \mathrm{~h}$ day. Minutes per $12 \mathrm{~h}$ day were calculated by multiplying the fraction of sedentary time (i.e. sedentary time/ wear time) by $720 \mathrm{~min}$ (i.e. $12 \mathrm{~h}$ ). Changes in sedentary time were calculated by subtracting sedentary time at baseline from sedentary time at follow up. As the current study did not include a 'gold-standard' of non-wear time, the manual data reduction using log sheets in combination with visual screening of the accelerometer data was used as reference method. This method is less prone to cause differences in sedentary time between age groups and has been used previously as a reference method [28]. Data were tested for normality and differences in sedentary time and the fragmentation of sedentary behavior between the four rules were examined using repeated measures ANOVA with Bonferroni post hoc tests.

\section{Results}

Participant characteristics are described in Table 1 . At 9 and 12 years of age, 592 and 508 participants received an accelerometer, resulting in 517 and 440 participants with valid measurements using the most restrictive zero criterion ( $\geq 10 \mathrm{~min}$ of consecutive zeros). Of the 517 children measured at baseline at age 9, 369 had valid measures at age 12 and were included in the analysis when looking at change in sedentary behavior.
Average sedentary time and changes in sedentary time are shown in Table 2. For the 517 participants included in the analysis at 9 years of age, mean sedentary time was found to be lowest when using the $10 \mathrm{~min}$ zero string rule (364 min per day) and highest using the 60 min zero string rule (426 min per day). Differences between methods were significant (Bonferroni post-hoc $p<0.05$ for all). For the 440 participants included at age 12 the difference between rules increased, with average sedentary times ranging from $424 \mathrm{~min}$ per day to $518 \mathrm{~min}$ per day for the $10 \mathrm{~min}$ and 60 min zero string rules, respectively. Differences in mean daily sedentary time were significant between all methods (Bonferroni post-hoc $p<0.05$ ) except the manual reduction and $20 \mathrm{~min}$ zero string rule (Bonferroni post-hoc $p=1.00)$. Mean changes in daily sedentary time between age 9 and age 12 (including 369 participants) ranged from $59 \mathrm{~min}$ when using the $10 \mathrm{~min}$ rule to $91 \mathrm{~min}$ when using the $60 \mathrm{~min}$ rule. Differences between methods were significant for all groups (Bonferroni post-hoc $p<0.05$ ) except the manual reduction and $60 \mathrm{~min}$ zero string rules (Bonferroni post-hoc $p=1.0$ ). When sedentary time was adjusted for wear time (i.e. using a $12 \mathrm{~h}$ day as described above) differences in mean daily sedentary time between rules decreased from $62 \mathrm{~min}$ to $27 \mathrm{~min}, 95 \mathrm{~min}$ to $32 \mathrm{~min}$ and $32 \mathrm{~min}$ to $9 \mathrm{~min}$ for age 9 , age 12 and mean changes in sedentary time between age 9 and 12, respectively.

As shown in Table 2, comparing the zero string rules to the manual rule (treated as a reference in this instance) resulted in significant differences between the manual rule and the 10, 20 and $60 \mathrm{~min}$ rules at age $9(p<0.05)$. The $10 \mathrm{~min}$ zero string rule resulted in the smallest error in mean sedentary time compared to the manual rule $(-9.5 \mathrm{~min})$ at age 9 . At age 12 , the 10 and $60 \mathrm{~min}$ rules resulted in significant under and overestimation of mean sedentary time, respectively, compared to the manual rule $(p<0.05)$. The 20 min zero string rule resulted in a small non-significant overestimation $(2.7 \mathrm{~min})$ of mean daily sedentary time compared to the manual rule. In addition, when looking at changes in sedentary time the 10 and $20 \mathrm{~min}$ rule underestimated mean change

Table 1 Participant characteristics at age 9 and $12 \mathrm{y}$

\begin{tabular}{|c|c|c|c|c|c|c|c|c|c|c|c|c|}
\hline & $9 y(n=5$ & $17)^{*}$ & & $12 y(n=$ & $440)^{* *}$ & & $9 y(n=$ & $69)^{* * *}$ & & $12 y(n=$ & $369)^{* * *}$ & \\
\hline & $\begin{array}{l}\text { All par } \\
\text { with v }\end{array}$ & $\begin{array}{l}\text { ipants } \\
\text { d data }\end{array}$ & & $\begin{array}{l}\text { All pa } \\
\text { with }\end{array}$ & $\begin{array}{l}\text { dants } \\
\text { data }\end{array}$ & & $\begin{array}{l}\text { Partic } \\
\text { data }\end{array}$ & ts $x$ & $\begin{array}{l}\mathrm{h} \text { valid } \\
\mathrm{y} \text { and } 12 \mathrm{y}\end{array}$ & $\begin{array}{l}\text { Partic } \\
\text { data }\end{array}$ & ths w & $\begin{array}{l}\text { valid } \\
12 y\end{array}$ \\
\hline & Mean & $\mathrm{SD}$ & Range & Mean & SD & Range & Mean & SD & Range & Mean & SD & Range \\
\hline Age (years) & 9.3 & 0.4 & $8.4-10.2$ & 12.5 & 0.3 & $11.6-13.3$ & 9.3 & 0.4 & $8.4-10.1$ & 12.5 & .3 & $11.6-13.3$ \\
\hline Height (cm) & 135.6 & 6.3 & $116.1-154.6$ & 154.6 & 7.8 & $129.5-176.2$ & 135.2 & 6.2 & $116.1-154.1$ & 154.7 & 7.7 & $129.5-174.9$ \\
\hline Weight (kg) & 33.4 & 7.5 & $19.2-70.8$ & 49.5 & 12.0 & $23.6-112$ & 33.0 & 7.2 & $19.2-67.1$ & 49.6 & 11.9 & $23.6-112$ \\
\hline $\mathrm{BMI}\left(\mathrm{kg} / \mathrm{m}^{2}\right)$ & 18.0 & 2.9 & $12.6-31.4$ & 20.5 & 3.8 & $12.7-36.7$ & 17.9 & 2.8 & $12.6-29.4$ & 20.6 & 3.8 & $12.7-36.7$ \\
\hline BMI-Z score & 0.56 & 1.1 & $-3.1-3.4$ & 0.68 & 1.2 & $-4.0-3.6$ & 0.51 & 1.1 & $-3.1-3.4$ & 0.68 & 1.2 & $-4.0-3.6$ \\
\hline
\end{tabular}

-*248 boys and 269 girls; **202 boys and 238 girls; ****168 boys and 201 girls 
Table 2 Wear time and sedentary time in min/day per non-wear time rule

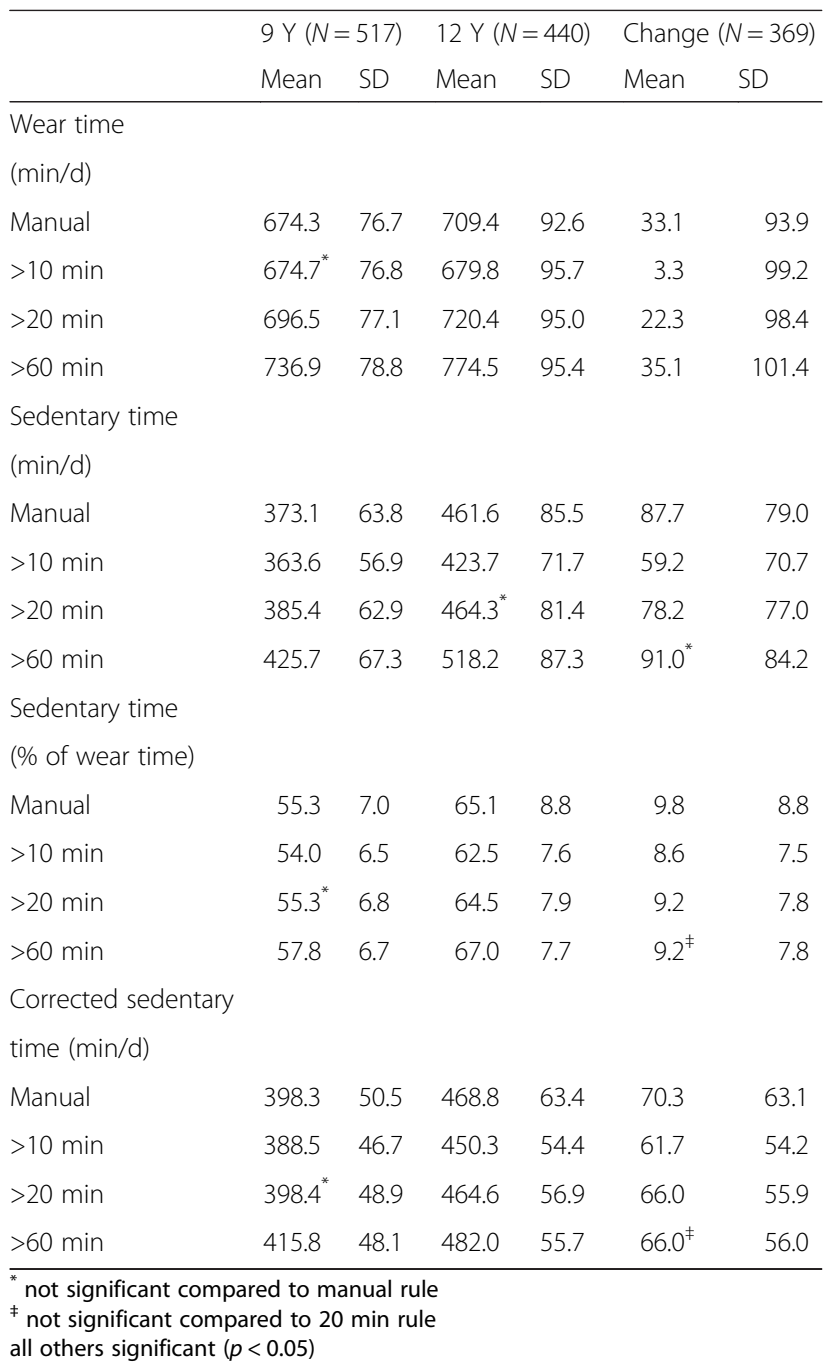

in sedentary time compared to the manual rule $(p<0.05)$. However, when using sedentary time corrected for wear time, the 20 and 60 min rules resulted in the closest estimates of change in sedentary time compared to sedentary time determined by the manual data reduction rule at both ages (Table 2).

\section{Discussion}

Analyses showed significant differences in sedentary time and changes in sedentary time between the different nonwear rules. At age 9 years, the $10 \mathrm{~min}$ zero string nonwear rule resulted in the closest estimates of sedentary time compared to the manual rule. However, when examining sedentary time at age 12 years and changes in sedentary time, $20 \mathrm{~min}$ and $60 \mathrm{~min}$ zero-string rule compared best to the manual rule, respectively. This finding is in line by a study done by Chinapaw et al. [12] who suggested 60 min of zero counts was the optimal non-wear time rule in children (mean age 11.7y). When correcting for wear time, the effect of the non-wear rule on sedentary time and changes in sedentary time appeared to decrease. The zero string rule of $20 \mathrm{~min}$ of consecutive zeros resulted in the closest estimates to the reference rule at both age 9 and 12 years. This finding is similar to that observed by Esliger et al. [29]. In their study $76 \%$ of $8-13$ year olds had zero string bouts greater than 10 min (mean $17.5 \mathrm{~min}$ ) suggesting a $20 \mathrm{~min}$ zero string rule might be appropriate to use in children. Looking at the difference between non-wear rules at both age groups, we noted that differences were larger at an older age. These findings are similar to those reported by Atkin et al. who found differences in sedentary time between non-wear rules ranged from $6 \mathrm{~min}$ to $30 \mathrm{~min}$ and from $18 \mathrm{~min}$ to $54 \mathrm{~min}$ at age 9 and age 15 , respectively [16].

The increase in differences between non-wear rules at an older age may indicate that the chosen length of zero string counts to define non-wear time becomes more significant at an older age. This is in line with some evidence on changes in patterns of sedentary behavior over time [20]. The changes in patterns of sedentary behavior (i.e. longer bouts of sitting as children or adolescents get older) might be a cause of the larger differences between non-wear rules at an older age. To investigate this further, we analyzed the changes in bouts of sedentary time from age 9 to age 12 (Table 3). These analyses showed an increase in longer bouts of sedentary behavior from age 9 to age 12. Therefore, when using shorter non-wear rules, longer bouts of sedentary behavior may be classified as non-wear time, leading to an underestimation of sedentary time.

In line with the findings at both age groups, changes in sedentary time were either underestimated (i.e. $10 \mathrm{~min}$ rule) or overestimated (i.e. 20 and 60 min rules) compared to the manual data reduction rule. However, even though the $60 \mathrm{~min}$ rule resulted in the largest overestimation of sedentary time at both ages, when examining changes over time, it appeared to provide the closest estimate compared to the manual rule. The difference between the manual and 60 min zero string rules is very similar between the two age groups considered (i.e. an overestimation of $53 \mathrm{~min}$ and $57 \mathrm{~min}$ per day respectively). This indicates that the

Table 3 Number of bouts of sedentary behavior per day at age 9 years and 12 years by bout length

\begin{tabular}{lcccc}
\hline & \multicolumn{2}{c}{$9 Y(N=390)$} & \multicolumn{2}{c}{$12 Y(N=390)$} \\
Bouts of sedentary behavior & Mean & SD & Mean & SD \\
\hline $5-9 \mathrm{~min}$ & 10.1 & 3.1 & 13.7 & 4.3 \\
$10-14 \mathrm{~min}$ & 2.4 & 1.3 & 3.7 & 1.7 \\
$15-29 \mathrm{~min}$ & 1.1 & 0.9 & 2.4 & 1.4 \\
$\geq 30 \mathrm{~min}$ & 0.2 & 0.3 & 0.5 & 0.6 \\
\hline
\end{tabular}

Difference between age 9 years and 12 years significant for all $(p<0.05)$ 
amount of non-wear time falsely classified as sitting time is similar at both age groups when using the $60 \mathrm{~min}$ rule. This suggests that when using shorter non-wear time rules some of the longer bouts of sedentary time (which appear to happen more often at a later age) are excluded as nonwear time whereas they remain included when using a 60 min rule resulting in closer estimates of change in sitting time when using the 60 min rule.

Differences between non-wear rules decreased significantly in both age groups when correcting for wear time. Results showed that using either of the zero string rules led to a smaller amount of error when compared to the manual rule. The most accurate estimates compared to the manual reference method were found when using the 20 min rule in both age groups. This suggests that correcting for wear time is a reasonable method to adjust for the overestimation of sedentary time which might occur when using a slightly longer zero string rule (i.e. $20 \mathrm{~min}$ ) at a younger age. However, the underestimation of sedentary time which occurred when using shorter rules appeared to be less affected by controlling for wear time. In addition, when using the corrected sedentary time, the $20 \mathrm{~min}$ and $60 \mathrm{~min}$ rules provided the most accurate estimates of changes over time. Therefore, when conducting longitudinal studies it may be worth using longer zero-string rules and correcting sedentary time for wear time. This may result in more accurate estimates of sedentary time as well as an improvement in the comparability between studies in future.

The present study had several strengths and limitations. The sample size and longitudinal aspect of the study are strengths. To our knowledge this is the first study examining the effect of non-wear rules on longitudinal data and the first to consider change in sedentary time, an important variable to intervention studies and longitudinal studies. In addition, the same data were used in each comparison, which controls for variables other than the nonwear definition. However, it has to be noted that while the $100 \mathrm{cpm}$ cut point provides the closest estimate to true sitting time [27] some standing may be included. This study did not have a 'gold-standard' or criterion measure of non-wear time, but manual data reduction using log sheets seems a reasonable reference method, and has been used as such previously [28]. Log sheets and accelerometer data were visually inspected by a trained researcher to reduce any potential measurement error related to the log sheets. In addition, the manual diary as a reference tool was unlikely to cause differences between age groups based on the rules used. This might have been the case if only the zero string rules were used. Also, this study adds to the literature as it indicates the difference between four commonly used non-wear criteria, even without using the manual criteria as a reference method. Several studies have been using algorithms which allow for interruptions of non-wear time. These were not included in the current study as it was decided to include some of the most commonly used and simple non-wear rules [13]. For the inclusion criteria for minimum wear time, it is possible that the results might be slightly different for different days of the week. However, most of the commonly used inclusion criteria for wear-time in the literature specify a certain amount of days without specifying which days should be included. Lastly, the study focused on two specific age groups and therefore generalizability to other age groups may be limited.

\section{Conclusion}

The present study has shown that using different rules to define non-wear time results in significant differences in sedentary time and changes in sedentary time. Whether these differences are large enough to be biologically or clinically significant may depend on the application. However, correcting for wear time appears to be a reasonable approach to limit these methodological differences and improve comparability between studies. Lastly, using a $20 \mathrm{~min}$ zero-string rule while correcting for wear time might be the method of choice when measuring sedentary time and changes in sedentary time in 9 and 12 year old children.

\section{Competing interests \\ The authors declare that they have no competing interests.}

\section{Authors' contributions}

$X J$ had the primary responsibility of analysis and interpretation of the data, and paper drafting. LB and JKR has been involved in the acquisition of the data and contributed to the interpretation of the data and paper drafting. $\mathrm{KP}, J \mathrm{JR}, \mathrm{AS}$ and MP collaboratively designed the research and each contributed to the interpretation of the data and paper drafting. All authors read and approved the final manuscript.

\section{Acknowledgements}

This work was supported by grants from the Scottish Government Chief Scientist Office (grant CZH/4/484 and CZH/4/979), the UK National Prevention Research Initiative, and Gateshead PCT. The cohort was first set up with funding from the Henry Smith Charity and Sport Aiding Medical Research for Kids. We appreciate the support of Gateshead Health National Health Service Foundation Trust, Gateshead Education Authority, and local schools. We thank members of the research team for their effort. We especially thank the families and children who participated in the Gateshead Millennium Study.

\section{Author details}

${ }^{1}$ School of Psychological Sciences and Health, University of Strathclyde, Glasgow, Scotland G1 1QN, UK. ${ }^{2}$ Institute of Health \& Society, Human Nutrition Research Centre, Newcastle University, Newcastle Upon Tyne, UK. ${ }^{3}$ Institute of Health \& Society Newcastle, Sir James Spence Institute of Child Health, Royal Victoria Infirmary, Newcastle University, Newcastle Upon Tyne, UK.

Received: 12 December 2014 Accepted: 18 May 2015

Published online: 23 May 2015

\section{References}

1. Sedentary Behaviour Research Network. Standardized use of the terms "sedentary" and "sedentary behaviours". Appl Physiol Nutr Metab. 2012;37:540-2 
2. Tremblay MS, LeBlanc AG, Kho ME, Saunders TJ, Larouche R, Colley RC, et al. Systematic review of sedentary behaviour and health indicators in school-aged children and youth. Int J Behav Nutr Phys Act. 2011;8:98.

3. Biddle SJ, O'Connell S, Braithwaite RE. Sedentary behaviour interventions in young people: a meta-analysis. Br J Sports Med. 2011;45:937-42.

4. Trost SG, Mclver KL, Pate RR. Conducting accelerometer-based activity assessments in field-based research. Med Sci Sports Exerc. 2005;37(Suppl):S531-43.

5. Heil DP, Brage S, Rothney MP. Modeling physical activity outcomes from wearable monitors. Med Sci Sports Exerc. 2012:44 Suppl 1:S50-60.

6. Trost SG, Loprinzi PD, Moore R, Pfeiffer KA. Comparison of accelerometer cut points for predicting activity intensity in youth. Med Sci Sports Exerc. 2011;43:1360-8.

7. Mattocks C, Ness A, Leary S, Tilling K, Blair SN, Shield J, et al. Use of accelerometers in a large field-based study of children: protocols, design issues, and effects on precision. J Phys Act Health. 2008;5 Suppl 1:S98-S111.

8. Corder K, Sharp SJ, Atkin AJ, Griffin SJ, Jones AP, Ekelund U, et al. Change in objectively measured physical activity during the transition to adolescence. Br J Sports Med. 2013. doi:10.1136/bjsports-2013-093190.

9. Kwon S, Burns TL, Levy SM, Janz KF. Breaks in sedentary time during childhood and adolescence: lowa Bone Development Study. Med Sci Sports Exerc. 2012;44:1075.

10. Ojiambo R, Cuthill R, Budd H, Konstabel K, Casajus JA, González-Agüero A, et al. Impact of methodological decisions on accelerometer outcome variables in young children. Int J Obes. 2011;35 Suppl 1:S98-S103.

11. Basterfield L, Adamson AJ, Parkinson KN, Maute U, Li P-X, Reilly JJ. Surveillance of physical activity in the UK is flawed: validation of the Health Survey for England Physical Activity Questionnaire. Arch Dis Child. 2008;93:1054-8.

12. Chinapaw MJM, de Niet M, Verloigne M, De Bourdeaudhuij I, Brug J, Altenburg TM. From sedentary time to sedentary patterns: accelerometer data reduction decisions in youth. PLoS One. 2014;9:e111205.

13. Cain KL, Sallis JF, Conway TL, Van Dyck D, Calhoon L. Using accelerometers in youth physical activity studies: a review of methods. J Phys Act Health. 2013;10:437-50.

14. Choi L, Liu Z, Matthews CE, Buchowski MS. Validation of accelerometer wear and nonwear time classification algorithm. Med Sci Sports Exerc. 2011;43:357-64.

15. Tanaka C, Reilly JJ, Huang WY. Longitudinal changes in objectively measured sedentary behaviour and their relationship with adiposity in children and adolescents: systematic review and evidence appraisal. Obes Rev. 2014;15:791-803.

16. Atkin AJ, Ekelund U, Møller NC, Froberg K, Sardinha LB, Andersen LB, et al. Sedentary time in children: influence of accelerometer processing on health relations. Med Sci Sports Exerc. 2012;45:1097-104.

17. Trang NH, Hong TK, van der Ploeg HP, Hardy LL, Kelly PJ, Dibley MJ. Longitudinal sedentary behavior changes in adolescents in Ho Chi Minh City. Am J Prev Med. 2013;44:223-30.

18. Mitchell JA, Pate RR, Dowda M, Mattocks C, Riddoch C, Ness AR, et al. A prospective study of sedentary behavior in a large cohort of youth. Med Sci Sports Exerc. 2012;44:1081-7.

19. Ortega FB, Konstabel K, Pasquali E, Ruiz JR, Hurtig-Wennlöf A, Mäestu J, et al. Objectively measured physical activity and sedentary time during childhood, adolescence and young adulthood: a cohort study. PLOS ONE 2013, 8:doi:10.1371/journal.pone.0060871.

20. Carson V, Cliff D, Janssen X, Okely AD. Longitudinal levels and bouts of sedentary time among adolescent girls. BMC Pediatr. 2013;13:173.

21. Parkinson KN, Pearce MS, Dale A, Reilly JJ, Drewett RF, Wright CM, et al. Cohort profile: the gateshead millennium study. Int J Epidemiol. 2011;40:308-17.

22. Cole TJ, Freeman JV, Preece MA. Body mass index reference curves for the UK, 1990. Arch Dis Child. 1995:73:25-9.

23. Trost SG. Objective measurement of physical activity in youth: current issues, future directions. Exerc Sport Sci Rev. 2001;29:32-6.

24. de Vries SI, Bakker I, Hopman-Rock M, Hirasing RA, van Mechelen W. Clinimetric review of motion sensors in children and adolescents. J Clin Epidemiol. 2006;59:670-80

25. Basterfield L, Adamson AJ, Frary JK, Parkinson KN, Pearce MS, Reilly JJ. Longitudinal study of physical activity and sedentary behavior in children. Pediatrics. 2011;127:e24-30.
26. Basterfield L, Adamson AJ, Pearce MS, Reilly JJ. Stability of habitual physical activity and sedentary behavior monitoring by accelerometry in 6-to 8-year-olds. J Phys Act Health. 2011:8:543-7.

27. Ridgers ND, Salmon J, Ridley K, O'Connell E, Arundell L, Timperio A. Agreement between activPAL and ActiGraph for assessing children's sedentary time. Int J Behav Nutr Phys Act. 2012;9:7.

28. Winkler EA, Gardiner PA, Clark BK, Matthews CE, Owen N, Healy GN. Identifying sedentary time using automated estimates of accelerometer wear time. Br J Sports Med. 2012;46:436-42.

29. Esliger DW, Copeland JL, Barnes JD, Tremblay MS. Standardizing and optimizing the use of accelerometer data for free-living physical activity monitoring. J Phys Act Health. 2005;2:366-83.

\section{Submit your next manuscript to BioMed Central and take full advantage of:}

- Convenient online submission

- Thorough peer review

- No space constraints or color figure charges

- Immediate publication on acceptance

- Inclusion in PubMed, CAS, Scopus and Google Scholar

- Research which is freely available for redistribution 